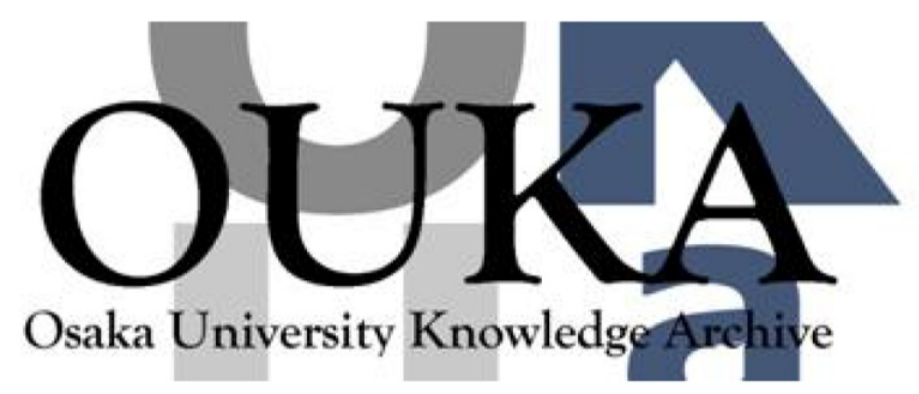

\begin{tabular}{|c|l|}
\hline Title & $\begin{array}{l}\text { Label-free ultrathin Pt film biosensor using } \\
\text { phonon vibrations excited by ultrafast light } \\
\text { pulses }\end{array}$ \\
\hline Author(s) & $\begin{array}{l}\text { Ogi, Hirotsugu; Matsumoto, Kozo; Fujita, Yusaku } \\
\text { et al. }\end{array}$ \\
\hline Citation & Applied Physics Express. 3(1) p. 017001 \\
\hline Issue Date & $2009-12-25$ \\
\hline oaire:version & AM \\
\hline URL & https://hdl. handle.net/11094/84154 \\
\hline rights & \\
\hline Note & \\
\hline
\end{tabular}

Osaka University Knowledge Archive : OUKA

https://ir. Library. osaka-u. ac. jp/

Osaka University 


\title{
Label-Free Ultrathin Pt Film Biosensor Using Phonon Vibrations Excited by Ultrafast Light Pulses
}

\author{
Hirotsugu Ogi, Kozo Matsumoto, Yusaku Fujita, Tetsuya Kawamoto, Nobutomo Nakamura, and Masahiko Hirao \\ Graduate School of Engineering Science, Osaka University, Toyonaka, Osaka 560-8531, Japan
}

\begin{abstract}
An ultrathin film oscillator biosensor is presented for highly sensitive monitoring of binding reactions among biomolecules in solutions. The phonon vibration along the thickness direction of an 18- $\mathrm{nm}$ Pt film is measured by ultrafast light pulses, whose fundamental resonance frequency is $120 \mathrm{GHz}$. The vibrational amplitude is monitored during binding reaction between human immunoglobulin $\mathrm{G}$ ( $\mathrm{hlg} \mathrm{G}$ ) and anti-hlgG antibody, which exponentially decreases as the reaction progresses. A $6 \mathrm{pM}$ hlgG solution can be detected. This biosensor also shows the ability to determine the affinity between proteins through the concentration dependence of the reaction rate.
\end{abstract}

Recent advances in biosensor technologies have established the label-free detection of target molecules in liquids. Especially, quartz-crystal-microbalance (QCM) biosensors ${ }^{1-3)}$ and surface-plasmon-resonance (SPR) biosensors ${ }^{4-6)}$ allow the real-time monitoring of binding reactions between receptors immobilized on the sensor chips and target analyte in liquids. The QCM biosensor recognizes adsorbed proteins on the quartz crystal through the change in the resonance frequency as well as in the damping factor of the vibration. The SPR biosensor monitors protein interactions through the change in the incident angle of the light used for the excitation of the surface plasmon wave, which is affected by the macroscopic electric properties in the evanescent field near the sensor surface. However, their sensitivity is usually lower than that of the other biosensors using labels such as enzyme-linked immunosorbent assay (ELISA) method. Thus, enhancement of the sensitivity of the label-free biosensors has been a central issue in the biophysical studies.

We here propose a highly sensitive label-free biosensor, focusing on the principle of the QCM biosensor. QCM biosensor is a mass sensitive biosensor (loading mass, ${ }^{7)}$ viscosity mass, ${ }^{8)}$ and coupling-water mass $\left.{ }^{9)}\right)$, and its sensitivity drastically increases with the decrease of the mass of the oscillator. Remaining the effective reaction area unchanged, the increase of the sensitivity can be achieved by the decrease of the thickness of the oscillator. Therefore, intensive efforts have been paid to decrease the oscillator thickness in QCM biosensors. ${ }^{10-13)}$ To date, the reported maximum frequency of the QCM biosensor is $170 \mathrm{MHz} .{ }^{14)}$ Further decrease in the crystal thickness is, however, never straightforward, because it deteriorates the robustness of the crystal.

We recently established the picosecond ultrasound spectroscopy for the study of elastic properties of ultrathin films. ${ }^{15,16)}$ Application of ultrafast light pulses onto the film causes high-frequency $(\sim 100 \mathrm{GHz})$ standingwave vibrations within the film, which have been understood as the $\Gamma$ points of the in-plane phonon vibration modes by the ab-initio calculation. ${ }^{17}$ ) Thus, using this phenomenon makes it possible to develop an ultrahighsensitive oscillator biosensor, because the thickness of the film oscillator $(\sim 20 \mathrm{~nm})$ is much smaller than the quartz plate used in conventional QCM biosensors $(\sim 100 \mu \mathrm{m})$. This paper reports the first application of the picosec-

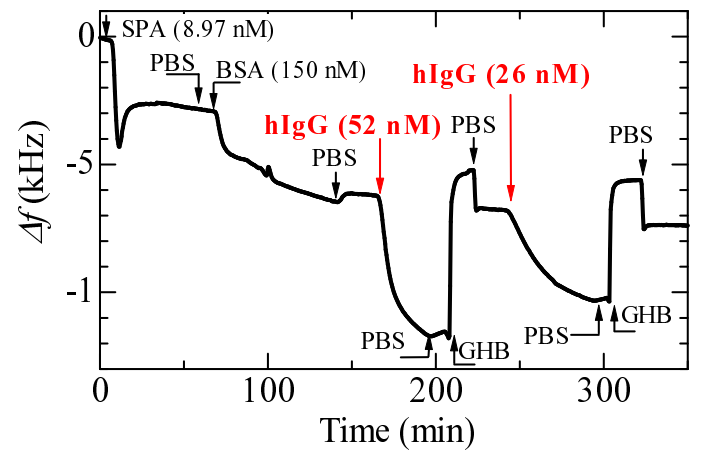

Fig. 1. Frequency change observed during the injection sequence of various solutions measured by the Pt-coated $55-\mathrm{MHz}$ QCM biosensors. ${ }^{19)}$ SPA was first immobilized on the surface-activated SAM, BSA was then injected for the blocking purpose, and after the PBS, the hIgG solution was injected for monitoring the specific binding between SPA and hIgG. The glycin $\mathrm{HCl}$ buffer (GHB) was used for dissociating hIgG molecules from SPA, making the repeatable measurement possible. Arrows indicate the arrival times of the solutions at the sensor cell.

ond ultrasound spectroscopy to the real-time monitoring of binding reaction between biomolecules, and demonstrates its high potential as a label-free biosensor.

As the film oscillator, we choose $\mathrm{Pt}$ for three reasons. (i) $\mathrm{Pt}$ is a suitable material for picosecond ultrasound spectroscopy ${ }^{15)}$ because it shows lower thermal conductivity, and the diffusion of electrons caused by the light pulse is restricted to the thin surface area, leading to the excitation of high-frequency phonons. ${ }^{18)}$ (ii)Sputtered Pt thin films show smooth surfaces $\left(R_{a}<0.5 \mathrm{~nm}\right)$, and (iii)they show high stiffness, ${ }^{15}$ ) indicating less defects and strong bonds between grains.

In order to use a Pt film as a biosensor, we need to immobilize a receptor protein on it through a linker material. However, few reports used $\mathrm{Pt}$ for biosensor surfaces and its applicability remains unclear. Au films have been used for this purpose, because they exhibit high binding affinity to thiol-terminated molecules, on which any proteins can be immobilized through a self-assembled monolayer (SAM). However, we failed to observe vibrations of $\mathrm{Au}$ films with a high $\mathrm{S} / \mathrm{N}$ ratio because of their high thermal conductivity. Therefore, we first investigated the applicability of $\mathrm{Pt}$ for the biosensor surface using a QCM measurement. Figure 1 shows the result, demonstrating that $\mathrm{Pt}$ shows high affinity to the thiol terminals and 


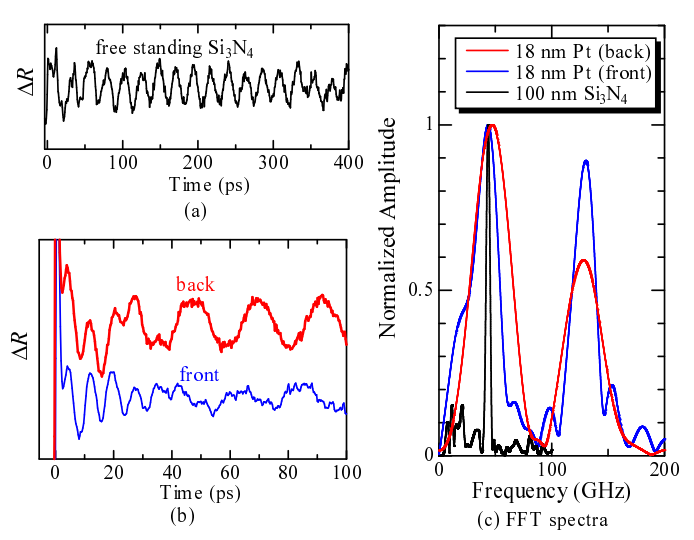

Fig. 2. Time-resolved reflectivity change, indicating longitudinal-wave vibrations of thin films, for (a) a 100-nm free-standing $\mathrm{Si}_{3} \mathrm{~N}_{4}$ film, (b) 18-nm Pt thin film on the $\mathrm{SiO}_{2}$ substrate when the light pulses entered from the $\mathrm{Pt}$ side (blue line), and that when the light pulses entered from the substrate side (red line). (c) Corresponding Fourier spectra.

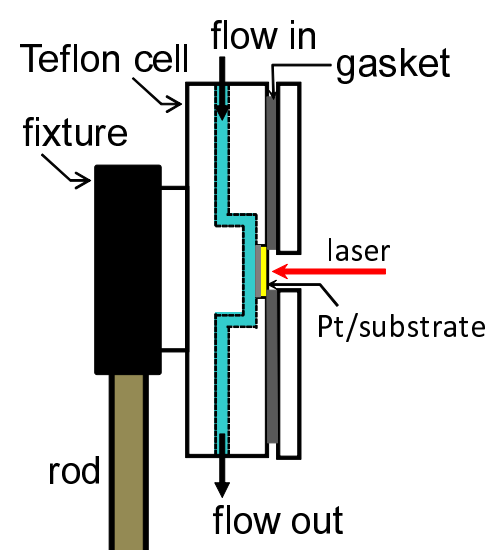

Fig. 3. Cross-section view of the sensor cell, where the $\mathrm{Pt} / \mathrm{SiO}_{2}$ chip is set so as to expose the Pt surface to the flow of the solution. The laser beams enter from the back side for the real-time monitoring of reactions on the Pt surface.

can be adopted as an effective biosensor surface: hIgG molecules were successfully detected by staphylococcus aureus protein A (SPA), which is known to specifically bind with $\mathrm{hIgG}$, immobilized on the Pt surface through the SAM. The thiol terminals of the SMA covalently bind with Pt. (The immobilization procedure of SPA and the measurement procedure of our QCM were the same as those used in the previous study. ${ }^{19}$ ) The sensing ability was identical to the case where Au surfaces were used.

We deposited an $18 \mathrm{~nm} \mathrm{Pt}$ thin film on an amorphous $\mathrm{SiO}_{2}$ substrate of 0.2 -mm-thick and 7 $\mathrm{mm}$ diameter by the magnetron-sputtering method: The substrate was cleaned in the piranha solution $\left(98 \% \mathrm{H}_{2} \mathrm{SO}_{4}: 33 \% \mathrm{H}_{2} \mathrm{O}_{2}=7: 3\right)$ before the deposition, and its surface was subjected to the pre-sputtering procedure for making tighter adhesion between the substrate and the Pt layer. The Pt thin film was then deposited.

Both pump and probe light pulses are perpendicularly focused on the sensor surfaces with an objective lens. Their spot diameters are about $20 \mu \mathrm{m}$ with the power of $\sim 5 \mathrm{~mW}$ for each. (Details of our optics ap- pear elsewhere. ${ }^{20)}$ ) Ultrahigh-frequency phonon modes are excited by the ultrafast light pulse, and they are detected by the delayed light pulse through the change in the reflectivity. ${ }^{21-24)}$ Thus, the time-resolved reflectivity change indicates vibrations of thin films. Figure 2 shows three measurements of standing wave vibrations for the Pt thin film and a $100-\mathrm{nm} \mathrm{Si}_{3} \mathrm{~N}_{4}$ free-standing film as a comparison. The $\mathrm{Si}_{3} \mathrm{~N}_{4}$ film was deposited on a $\mathrm{Si}$ substrate by a chemical-vapor deposition method, and a part of the substrate was removed by the wet-etching procedure with a potassium-hydroxide solution. A $10 \mathrm{~nm} \mathrm{Al}$ thin film was deposited as a ultrasound generator on one side of the remaining free-standing area, which was irradiated with the light pulses to measure vibrations of the $\mathrm{Si}_{3} \mathrm{~N}_{4}$ film. The fundamental longitudinal-wave vibration $(\sim 50 \mathrm{GHz})$ continues for a long time $(\sim 1 \mathrm{~ns})$ in this case [Fig. 2(a)].

The other two cases are measurements for the same $\mathrm{Pt} / \mathrm{SiO}_{2}$ sensor chip [Fig. 2(b)]. When the Pt film is irradiated from the front surface, the high-frequency vibration first occurs, which is followed by the lower frequency vibrations [blue line in Fig. 2(b)]. The former is the longitudinal-wave resonance within $\mathrm{Pt}$ and the latter is the Brillouin oscillation in the $\mathrm{SiO}_{2}$ substrate. ${ }^{24-26)}$ Because of the energy loss into the substrate, the standing wave attenuates more rapidly than the free-standing film case. (This indicates that removing the substrate in the measured area, we can increase the $\mathrm{Q}$ value of the vibration, which contributes to improve the stability of the response. In the future study, we will do this task.) When the $\mathrm{Pt}$ film is irradiated from the back surface (i.e., from the substrate side), the Brillouin oscillation becomes significant. (The front Pt surface was exposed to the solution as shown in Fig. 3 in this case.) However, we can still clearly observe the high-frequency oscillation after the excitation [red line in Fig. 2(b)], and its Fourier spectrum shows the same resonance frequency of the longitudinal wave $(\sim 120 \mathrm{GHz})$ as the front-excitation case [Fig. 2(c)]. In the immunosensor measurement, the Pt surface must be contacted with the solution, and we measure the phonon response from the substrate surface as shown in Fig. 3.

Surface modification was performed as follows. The Pt surface was cleaned in the piranha solution. It was rinsed with ultrapure water several times, and the sensor chip was immersed in a SAM (10 mM 10carboxy-1-decanethiol/absolute ethanol solution) for 20 $\mathrm{h}$ at $4^{\circ} \mathrm{C}$. After rinsing with absolute ethanol three times, it was immersed in a 100-mM EDC (1-ethyl-3-(3dimethylaminopropyl)carbodiimide, hydrochloride) solution for $1 \mathrm{~h}$ to activate the carboxyl terminals, and in the phosphate buffer solution (PBS, ph 7.4) containing $1 \mathrm{mg} / \mathrm{ml}$ anti-hIgG antibody and $100 \mu \mathrm{M} 100 \mathrm{mM}$ sulfoNHS (N-hydroxysulfosuccinimide sodium salt) for $10 \mathrm{~h}$ at room temperature. The remaining activated ester sites were blocked with a $10 \mathrm{mg} / \mathrm{ml}$ bovine-serum-albumin (BSA)/PBS solution.

The surface-modified sensor chip is set in the sensor cell shown in Fig. 3. The carrier solution of PBS flows along the Pt surface with the flow rate of $500 \mu \mathrm{l} / \mathrm{min}$. The back surface of the substrate is exposed to atmosphere, 


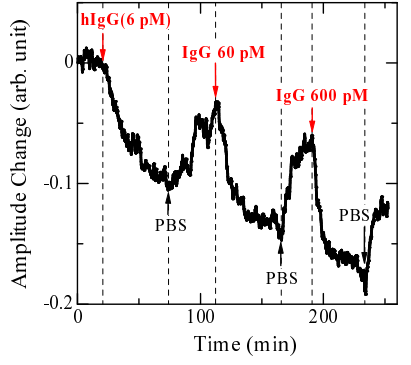

(a)

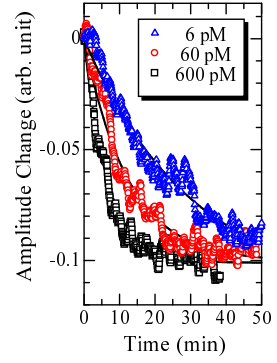

(b)
Fig. 4. (a)The phonon vibration amplitude at $30 \mathrm{ps}$ during the association and dissociation reactions between anti-hIgG antibody and hIgG. Arrows indicate the arrival times of the solutions at the sensor cell. (b) Binding curves and fitted exponential functions (solid lines) for the different hIgG-concentration solutions.

from which the light pulses enter. We used a homebuilt flow-injection system ${ }^{14)}$ for a steady measurement during the injection sequence.

For determining the resonance frequency, the reflectivity has to be measured in a wide delay-time range, but it takes a long time $(\sim 5 \mathrm{~min})$. Instead, we monitored the reflectivity at a fixed time of $30 \mathrm{ps}$ in Fig. 2(b), focusing on the vibrational amplitude change during association and dissociation reactions between anti-hIgG antibody and hIgG. We prepared the hIgG solutions by dissolving the lyophilized hIgG reagent (Athens Research and Technology, Inc.; product num. 16-16-090707; purity 95\%; IgG amount $10 \mathrm{mg}$ ) in PBS to make a $1 \mathrm{mg} / \mathrm{ml}$ solution, and by diluting it into PBS to obtain the intended concentrations.

The result is shown in Fig. 4(a). The vibration amplitude decreases as the biding reaction proceeds. We attribute this change to the viscosity-loading effect and the coupled-water loading effect rather than the frequency change, because we failed to observe significant frequency change between before and after the binding reaction completed. The arrival of the carrier PBS makes the phonon response recover, indicating the dissociation of hIgG molecules from anti-hIgG antibody. However, because of the incomplete dissociation by the PBS flow, we failed to see the dependence of the amount of the acoustic response on the hIgG concentration. Baseline stability was worse compared with a conventional QCM measurement, but the present biosensor can detect a low concentration analyte $(\sim 6 \mathrm{pM})$ clearly. Considering that the detection limit of conventional QCM and SPR biosensors for an antibody is of the order of $100 \mathrm{pM},{ }^{27-29)}$ the high-sensitivity potential involved in the present biosensor system is remarkable.

The phonon response during the binding reaction appears to obey the exponential law, and its change rate (exponential coefficient: $\alpha$ ) increases with the increase of the concentration of $\mathrm{hIgG}, C_{A}$. This is a typical behavior observed in a pseudo-first-order reaction. Assuming a linear relationship between the measured response and the adsorbed quantity, the exponential coefficient is proportional to the concentration of the analyte as $\alpha=k_{a} C_{A}+k_{d},{ }^{30)}$ where $k_{a}$ and $k_{d}$ denote the association and dissociation rate constants of the binding reaction, respectively. Thus, plotting $\alpha$ vs. $C_{A}$ provides the thermodynamics constants and then the equilibrium constant $K_{A}=k_{a} / k_{d}$ of the reaction. We fitted exponential functions to the responses during the binding reactions as shown in Fig. 4(b) and extracted the $K_{A}$ value as $K_{A}=1.02 \times 10^{9} \mathrm{M}^{-1}$. This value is consistent with typical binding affinity between antigen-antibody reactions, ${ }^{19)}$ supporting that the change in the acoustic response is caused by the binding reaction between the biomolecules.

In summary, we developed the label-free picosecondultrasound biosensor using an 18-nm Pt-film oscillator. The QCM measurement confirmed that the Pt thin film showed high affinity for the thiol-terminated linkers, on which receptor proteins were effectively immobilized. The binding reactions between anti-hIgG antibody and hIgG were monitored by the vibrational amplitude of 120 $\mathrm{GHz}$ standing-wave vibration of the ultrathin $\mathrm{Pt}$ film. The vibrational response clearly detected the $6 \mathrm{pM}$ hIgG solution. Although the detection mechanism is not completely identified, it will be associated with the viscosity and water-coupling effects. The decay rate of the phonon response depended on the analyte concentration, from which the equilibrium affinity between the proteins were determined. Thus, the present biosensor is also applicable to an affinity sensor. The future work should include improvements of the stability by making a standing-free $\mathrm{Pt}$ film as well as detailed study for the detection mechanisms.

1) H. Muramatsu et al.: Anal. Chem. 59 (1987) 2760.

2) Y. Liu et al.: Biosens. Bioelectron. 19 (2003) 9.

3) H. Ogi et al.: Anal. Chem. 78 (2006) 6903.

4) R. Kurita et al.: Anal. Chem. 79 (2007) 9572.

5) D. Nedelkov: Anal. Chem. 79 (2007) 5987.

6) X. Hoaa et al.: Biosens. Bioelectron. 23 (2007) 151.

7) G. Sauerbrey: Z. Phys. 155 (1959) 206.

8) K. Kanazawa and J. Gordon: Anal. Chim. Acta 175 (1985) 99.

9) P. Bingen et al.: Anal. Chem. 80 (2008) 8880.

10) M. Natesan et al.: Anal. Chem. 81 (2009) 3896.

11) H. Furusawa et al.: Anal. Chem. 81 (2009) 2268.

12) E. Uttenthaler et al.: Biosens. Bioelectron. 16 (2001) 735.

13) H. Ogi et al.: Jpn. J. Appl. Phys. 47 (2008) 4021.

14) H. Ogi et al.: Anal. Chem. 81 (2009) 8068.

15) H. Ogi et al.: Phys. Rev. Lett. 98 (2007) 195503.

16) H. Ogi et al.: Appl. Phys. Express 2 (2009) 105001.

17) H. Tanei et al.: Appl. Phys. Lett. 95 (2009) 011902.

18) G. Tas and H. Maris: Phys. Rev. B 49 (1994) 15046.

19) H. Ogi et al.: Anal. Chem. 80 (2008) 5494.

20) H. Ogi et al.: Appl. Phys. Lett. 90 (2007) 191906.

21) C. Thomsen et al.: Phys. Rev. Lett. 53 (1984) 989.

22) D. H. Hurley and O. B. Wright: Opt. Lett. 24 (1999) 1305.

23) O. Matsuda et al.: Phys. Rev. Lett. 93 (2004) 095501.

24) A. Devos and R. Côte: Phys. Rev. B 70 (2004) 125208.

25) C. Thomsen, et al.: Phys. Rev. B 34 (1986) 4129.

26) H. Ogi et al.: Phys. Rev. B 78 (2008) 134204.

27) N. Pan and J. Shih: Sens. Actuators B 98 (2004) 180.

$28)$ B. D. Spangler et al: J. Anal. Chim. Acta 444 (2001) 149.

29) L. Laricchia-Robbio and R. Revoltella: Biosens. Bioelectron. 19 (2004) 1753.

30) M. Eddowes: Biosensors 3 (1987) 1. 